\title{
THE
}

\section{Microscale Quantification of the Absorption by Dissolved and Particulate Material in Coastal Waters with an ac-9}

\author{
Michael S. Twardowski \\ James M. Sullivan \\ University of Rhode Island \\ Percy L. Donaghay \\ University of Rhode Island, donaghay@uri.edu \\ J. Ronald V. Zaneveld
}

Follow this and additional works at: https://digitalcommons.uri.edu/gsofacpubs

\begin{abstract}
Citation/Publisher Attribution
Twardowski, M.S., Sullivan, J. M., Donaghay, P. L., \& Zaneveld, J. R. (1999). Microscale Quantification of the Absorption by Dissolved and Particulate Material in Coastal Waters with an ac-9. J. Atmos. Oceanic Technol., 16, 691-707. doi: 10.1175/1520-0426(1999)0162.0.CO;2

Available at: https://doi.org/10.1175/1520-0426(1999)016<0691:MQOTAB>2.0.C0;2
\end{abstract}

This Article is brought to you for free and open access by the Graduate School of Oceanography at DigitalCommons@URI. It has been accepted for inclusion in Graduate School of Oceanography Faculty Publications by an authorized administrator of DigitalCommons@URI. For more information, please contact digitalcommons-group@uri.edu. 


\title{
Microscale Quantification of the Absorption by Dissolved and Particulate Material in Coastal Waters with an ac-9
}

\author{
Michael S. Twardowski \\ College of Oceanic and Atmospheric Sciences, Oregon State University, Corvallis, Oregon \\ James M. Sullivan And Percy L. Donaghay \\ Graduate School of Oceanography, University of Rhode Island, Narragansett, Rhode Island \\ J. RonaLd V. ZANEVELD \\ College of Oceanic and Atmospheric Sciences, Oregon State University, Corvallis, Oregon
}

(Manuscript received 10 October 1997, in final form 9 September 1998)

\begin{abstract}
Measuring coastal and oceanic absorption coefficients of dissolved and particulate matter in the visible domain usually requires a methodology for amplifying the natural signal because conventional spectrophotometers lack the necessary sensitivity. The WET Labs ac-9 is a recently developed in situ absorption and attenuation meter with a precision better than $\pm 0.001 \mathrm{~m}^{-1}$ in the raw signal, which is sufficient to make these measurements in pristine samples. Whereas the superior sensitivity of the ac- 9 has been well documented, the accuracy of in situ measurements for bio-optical applications has not been rigorously evaluated.

Obtaining accurate results with an ac-9 requires careful attention to calibration procedures because baselines drift as a result of the changing optical properties of several ac- 9 components. To correct in situ measurements for instrument drift, a pressurized flow procedure was developed for calibrating an ac- 9 with optically clean water. In situ, micro- $(\mathrm{cm})$ to fine- $(\mathrm{m})$ scale vertical profiles of spectral total absorption, $a_{t}(\lambda)$, and spectral absorption of dissolved materials, $a_{g}(\lambda)$, were then measured concurrently using multiple meters, corrected for drift, temperature, salinity, and scattering errors and subsequently compared. Particulate absorption, $a_{p}(\lambda)$, was obtained from $a_{t}(\lambda)-a_{g}(\lambda)$. CTD microstructure was simultaneously recorded. Vertical profiles of $a_{g}(\lambda), a_{t}(\lambda)$, and $a_{p}(\lambda)$ were replicated with different meters within $\pm 0.005 \mathrm{~m}^{-1}$, and spectral relationships compared well with laboratory measurements and hydrographic structure.
\end{abstract}

\section{Introduction}

Many compositional analyses of seawater require a precision of $\pm 0.005 \mathrm{~m}^{-1}$ or better in absorption coefficients through the visible domain. These analyses include using absorption to estimate coastal and oceanic pigment concentrations, chromophoric dissolved organic matter (CDOM; also Gelbstoff or gilvin) concentrations, and variations in CDOM composition. The need for high sensitivity is often magnified when a computation involving two or more coefficients is necessary to obtain a desired parameter. Error in each coefficient propagates through the calculation, increasing the uncertainty in the final result. Examples include calculating particulate absorption by subtracting CDOM ab-

Corresponding author address: Dr. Michael Twardowski, COAS Oregon State University, 104 Ocean Admin. Bldg., Corvallis, OR 97331

E-mail: mtwardo@oce.orst.edu sorption from total absorption, taking particulate absorption ratios, and spectral analyses of CDOM absorption.

Consequently, the evaluation of particulate and CDOM absorption coefficients have required specialized methods to amplify the natural signal because commercial spectrophotometers lacked the necessary sensitivity. Conventional spectrophotometers also are not equipped to collect scattered light from a sample with suspended particles. Particulate absorption coefficients have therefore been estimated with variations of the filter pad technique (Yentsch 1957, 1960, 1962; Mitchell and Kiefer 1988) and other methods such as microphotometry, where the optical and geometric properties of individual particles in an assemblage are determined microscopically (Iturriaga and Siegel 1989). Low coastal and oceanic CDOM absorption coefficients have been measured by using spectrophotometers modified to accommodate very long pathlength flow cells (Bricaud et al. 1981) or by using hydrophobic organic resins to 
extract the dissolved humic substances in seawater, producing a concentrated solution with a detectable absorption signal when eluted (Carder et al. 1989).

Despite the utility of these methods, they have limitations. Assumptions are usually required in order to reconstruct the desired inherent optical property (IOP) in the original sample. For example, a pathlength amplification factor must be defined in the fiber pad technique to correct for increases in the light path due to scattering by the filter with embedded sample (Mitchell and Kiefer 1988). Also, in the extraction and reconstitution of aquatic humus (Carder et al. 1989), assumptions must be made on the consistency of the optical character of the substance through various chemical manipulations (e.g., acidification, hydrophobic extraction, reconstitution in freshwater, freeze-drying, $\mathrm{pH}$ adjustments). Indeterminate errors are therefore unavoidable in the application of these methods. Additionally, the methodology can be laborious, requiring discrete sampling and sample storage in most cases.

The ac-9 is a nine-wavelength in situ spectral absorption and attenuation meter developed by WET Labs Inc., Philomath, Oregon (Moore et al. 1992; Zaneveld et al. 1992; Moore 1994), which addresses the problems of insufficient sensitivity in conventional spectrophotometers and the requirement of collecting discrete samples. The instrument has dual, $25-\mathrm{cm}$ pathlength flow tubes and allows real-time measurements in a profiling, moored, or towed deployment. Total absorption, $a_{t}(\lambda)$, and attenuation, $c_{t}(\lambda)$, coefficients in the visible domain can be measured in unconcentrated, natural samples (Zaneveld et al. 1992), and spectral CDOM absorption coefficients, $a_{g}(\lambda)$, can be obtained by attaching a $0.2-\mu \mathrm{m}$ prefilter at the intake (Twardowski et al. 1996; Twardowski 1998). Total particulate absorption (including living cells and detritus), $a_{p}(\lambda)$, is obtained by subtracting $a_{g}$ from $a_{t}$. With a sampling rate of about $6 \mathrm{~Hz}$, a typical $50-\mathrm{m}$ profile will produce the equivalent of $3000 a$ and $c$ spectra in less than 10 min, making this method of measurement potentially very powerful.

The absolute accuracy of ac-9 measurements, however, is defined by how precisely the instrument can be calibrated to the absorption of optically pure water and the accuracy of corrections that must be considered when measuring absorption at low levels. Although reproducible results may be obtained with an ac-9, this fact alone does not guarantee the results are accurate. Unlike bench-top dual-beam spectrophotometers, there is no new reference blank simultaneously subtracted from in situ measurements with the ac-9. Therefore, instrument drifts from changing filter and detector characteristics, and slight shifts in the alignment and transmissivity of the optics are not inherently accounted for.

It is now widely recognized that ac-9s do in fact drift over time. At any given time, this bias error can easily be more than an order of magnitude greater than the precision of the measurement. An ac-9 is supplied from WET Labs already calibrated to zero for pure water, and drift is represented as a deviation from zero when pure water is passed through the flow cells of the instrument. This drift introduces an error in the absolute absorption values and their relative spectral relationship. Measurements that are uncorrected for drift, especially in blue water regions, will often result in negative values for absorption and inevitably make little sense. Consequently, there has been a need for calibration methods to account for these drifts that can be implemented in the field.

We have developed a pressurized flow method for calibrating the ac-9 in the laboratory and in the field. One of the goals of this work was to accurately quantify drift in several ac-9s, remove the bias errors, and critically evaluate and intercompare the results. Guidelines for proper use in the field and the basic steps required to obtain accurate results are assessed. A spectral correction for salinity to account for the refractive index differences between saltwater and freshwater is measured using an extensively purified sea salts solution. The effectiveness of applying these methods to measure $a_{g}(\lambda), a_{t}(\lambda), a_{p}(\lambda)$, and the spectral properties of $a_{g}(\lambda)$ and $a_{p}(\lambda)$ with the ac-9 is evaluated by comparing replicate measurements between different meters. The coefficients $a_{g}(\lambda)$ measured by an ac-9 and commercial spectrophotometers are also compared.

\section{Methodology}

a. ac-9 description

The ac-9 consists of dual, $25-\mathrm{cm}$ pathlength flow tubes with each path having an independent incandescent light source, detector, and reference detector (WET Labs 1997). Absorption (a) and attenuation (c) channels share nine 10-nm full-width, half-maximum interference filters (usually a subset of the nominal wavelengths $412,440,488,520,532,555,560,630,650,676,715$, and $750 \mathrm{~nm}$ ) imbedded in a rotating wheel impinging the incident beam. Light from the source passes through an interference filter and is then split into a reference beam, $I_{o}$, and a primary beam, $I$, which passes through the sample. Absorption and attenuation are calculated by the supplied acquisition software, WETView, from the negative natural $\log$ of the transmission $\left(I / I_{o}\right)$ divided by the pathlength, $0.25 \mathrm{~m}$. The sampling rate is approximately $6 \mathrm{~Hz}$. The absorption flow cell is a reflective quartz tube design with diffuser described by Zaneveld and Bartz (1984) and implemented in the laboratory by Zaneveld et al. (1990). Forward scattered light out to $41^{\circ}$ from the incident ray is included in the absorption measurement.

The original ac-9 pure water offsets, supplied with the instrument, are determined from water calibrations performed at WET Labs. These water offsets are contained in an instrument-specific [.dev] file that WETView applies by subtraction to all in situ measurements. After the instrument is calibrated for the absorption of 
pure water at WET Labs, it is dried and dry nitrogen is passed through to obtain air calibration values. Air calibrations are supplied in an accompanying calibration record.

WETView also corrects for the effects that changes in internal temperature have on the optical performance of certain ac-9 components. For each wavelength, the correction is assumed to be linear with changing internal temperature, where the coefficient $K_{t}$ is the slope. The values of $K_{t}$ are determined at WET Labs and are contained in the [.dev] file. The maximum allowed deviation from the linear fit is $0.010 \mathrm{~m}^{-1}$ (D. Hankins, WET Labs, 1995, personal communication), and each channel has a unique standard deviation around the linear fit over the $5^{\circ}-40^{\circ} \mathrm{C}$ temperature range tested. The most robust fit usually occurs below $32^{\circ}-35^{\circ} \mathrm{C}$ internal temperature, and it is therefore recommended that the ac9 not be used when internal temperatures rise above about $32^{\circ} \mathrm{C}$.

\section{b. Calibrations}

A requirement for accurate ac- 9 calibrations is that optically clean water be used, which is highly reproducible over long periods of time. If the water has changing optical properties, then this will falsely manifest itself as a drift in the ac-9. The calibration setup and execution must also be conducted such that contamination of the clean water is minimized. All water for calibrations was supplied by a four-cartridge Barnstead Nanopure system, producing water of $18 \pm 0.1$ $\mathrm{M} \Omega \mathrm{cm}^{-1}$ ion purity. The water feed into the Nanopure system was pretreated with a Culligan deionizer. The water produced from the Nanopure system was filtered with a $0.2-\mu \mathrm{m}$ nylon filter (Barnstead) and dispensed into acid-cleaned 8- or 20-L polycarbonate carboys used in the calibrations. The water was allowed to degas at least $2 \mathrm{~h}$ before a calibration. For water calibrations in the field, the water purification system was brought to the field, and stored deionized water was used as a source to produce water of consistent quality and to ensure the system operated efficiently.

The ac-9 pressure windows were cleaned with ethanol and dried with lens paper before water and air calibrations and field measurements. We obtained better replication between water calibrations when the flow cells and pressure windows were dried with lens paper before each measurement. Eliminating bubbles was more difficult with drying droplets of water remaining in the flow cells. The water calibration setup is shown in Fig. 1.

Trapped air bubbles in the ac-9 flow cells were removed by opening the flow control clamp, increasing the flow rate to about $500 \mathrm{~mL} \mathrm{~min}^{-1}$, and gently tapping and rocking the ac- 9 and associated tubing. When the flow cells were free of bubbles (no spiking in data stream), the flow rate was restricted to less than $100 \mathrm{~mL}$ $\min ^{-1}$, pressurizing the flow cells to prevent cavitation from occurring during the calibration.

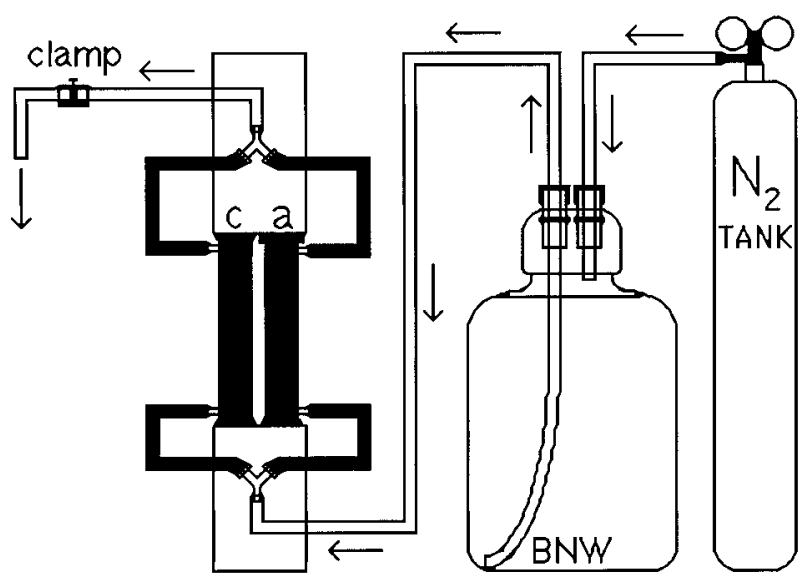

FIG. 1. Pure water calibrations for the ac-9. Nitrogen pressure is used to push Barnstead Nanopure Water (BNW) through the $a$ and $c$ flow cells of the ac-9. A clamp on the outflow is used to restrict the flow, pressurizing the flow cells to prevent cavitation. All tubing in contact with BNW before the measurement was Teflon. The carboy was polycarbonate. Polypropylene and polyethylene carboys should not be used because they leach materials into the stored water.

Calibration values were obtained by averaging absorption and attenuation coefficients over a period of at least $60 \mathrm{~s}$. The temperature of the outflow water was measured for temperature-dependent water absorption corrections [see section $2 \mathrm{e}(1)$ ]. The entire process was repeated until replicate calibration values for each wavelength in each channel varied less than approximately $0.002 \mathrm{~m}^{-1}$. Replication was important in calibrations because stable baselines can be obtained with contaminated (e.g., bubbles) flow cells. The final calibration values represent water offset adjustments, or drift corrections, that must be applied to subsequent measurements. They can be applied in postprocessing, as we have done for the data in this work, or by updating the water offset constants in the ac-9 [.dev] file before a measurement is taken. Not altering [.dev] files allowed us to accumulate records of water offset drifts over time and also provided flexibility in the application of water offset adjustments.

\section{c. Laboratory absorption measurements}

Offset drifts were obtained from water calibrations before any measurements. For particle-free samples or samples passing through an inline filter, the calibration setup described previously was used with the sample replacing the clean water in the pressurized carboy.

The calibration setup was problematic, however, with a particle-containing sample because of particle sorting when the flow was split by a "Y." While running unialgal cultures through the ac-9 in the lab with a "Y," we found that restricting flow rates under pressure can cause an increase in absorption or attenuation in one channel and a simultaneous decrease in the other channel (Fig. 2). One possible explanation is that particles 




FIG. 2. Particle sorting through a "Y" when the flow is restricted; FO (flow opened), FR (flow restricted). The absorption channel shows an increase, and the attenuation channel shows a simultaneous decrease when the clamp on the outflow is partially closed. When the clamp is opened, baselines values are restored. The sample was a culture of Asterionellopsis sp.

are experiencing a turbulence-related sorting preferentially into one or the other flow cells. A change in flow rates alone between the two flow cells would not be expected to give this result because the concentration of particles should be constant in both channels regardless of flow rate. The pressurized carboy system was therefore modified so that individual intake tubes could feed each flow cell. Two lengths of Teflon tubing were run from the bottom of the pressurized carboy, through compression fittings in the cap, to their respective flow cell inlets. A third compression fitting in the cap was for nitrogen gas input. With this setup, no changes in mean absorption or attenuation were observed by restricting flow rate in either cell.

To compare the ac- 9 output with results obtained using commercial spectrophotometers, $a_{g}(\lambda)$ was measured for a dilute coffee extract with a Hewlett-Packard 8452 diode-array spectrophotometer (HP8452), a Shimadzu UV-260 scanning, dual-beam spectrophotometer (SUV-260), and an ac-9. Absorbance, $A(\lambda)$, was measured by the spectrophotometers from $400-750 \mathrm{~nm}$ with $2-\mathrm{nm}$ bandwidth resolution in $10-\mathrm{cm}$ pathlength quartz cells with a sample volume of $25 \mathrm{~mL}$. Then $a_{g}(\lambda)$ was obtained from $A(\lambda)$ using

$$
a_{g}=2.303 \mathrm{~A} / \ell,
$$

where $\ell$ was the pathlength (meters) and 2.303 was a scaling constant converting logarithm base 10 to a nat- ural logarithm. The estimated precisions of the HP8452 and the SUV-260 were \pm 0.05 and $\pm 0.01 \mathrm{~m}^{-1}$, respectively.

The coffee sample was prepared by diluting brewed coffee to 5 ppt with Nanopure water. Nanopure water was used as a blank in the measurement. Since separate $10-\mathrm{cm}$ cells were used for the sample and the blank with the HP8452, both cells were filled with Nanopure and a sample-cell spectral offset was measured and subtracted from all the spectra afterward. To avoid errors in absorption between the sample and blank cells that arise due to temperature differences, both the sample and blank were kept at room temperature.

Samples were initially filtered using Whatman GF/F glass-fiber filters (e.g., Bricaud et al. 1981 used GF/C glass-fiber filters), but fibers from the filter were found to contaminate the filtrate. The scattered radiation from the suspended fibers was falsely read as absorbed radiation by the spectrophotometer. The resulting error in $a$ was $0.12 \mathrm{~m}^{-1}$ at $400 \mathrm{~nm}$, decreasing to about 0.05 $\mathrm{m}^{-1}$ at wavelengths greater than $600 \mathrm{~nm}$. The scattering error in the 400-500-nm range was typical of coastal shelf values for $a_{g}(\lambda)$ reported in the literature (e.g., Kirk 1994). As a result, samples discussed here were filtered through either a $0.2-\mu \mathrm{m}$ polycarbonate membrane filter (Nuclepore) or a $0.2-\mu$ m nylon capsule filter (Gelman) immediately prior to measurement. These filters were found not to contaminate samples.

\section{d. In situ absorption measurements}

The terms $a_{g}(\lambda)$ and $a_{t}(\lambda)$ were recorded with calibrated ac-9s during several periods of study in East Sound, Washington. East Sound is a productive fjord within the San Juan Islands, north of Puget Sound. The average depth is $30 \mathrm{~m}$, with a partial sill at the southern end about $14 \mathrm{~m}$ in depth in the center. Periodic stratification is common in the spring and summer when layers and patchy distributions of plankton can often be observed (Donaghay et al. 1996).

Before the cruises, the entire attenuation optical path of one ac-9, including the housing optics, was replaced with a second absorption optical path by WET Labs, resulting in a dual-channel absorption meter, termed an aa-9 (aa90106). Two separate, identical, absorption channels, the "aa" and "ab" channels, sharing interference filters and control and data acquisition electronics, allowed us to address the issue of replication and identify sources of instrument error.

\section{1) Microscale PROFILER}

An ac-9 and the aa-9 were positioned vertically in a deployment cage and secured with hose clamps. Large hose clamps were clamped around both ends of the meters, and smaller hose clamps were used to secure the large hose clamps to the cage. This mounting design minimized any torque on the meters. Water calibrations 
were performed on the meters after they were secured in the cage. After submersing the package and supplying power, the meters were allowed to warm up for 5-15 min to stabilize internal temperature. Warm-up time was a function of the particular ac-9 used, and both the temperature of the ambient water and air before deployment.

The profiling package consisted of a Seabird 911-plus CTD with fast response thermistor, $\mathrm{pH}$, and oxygen sensors, an ac-9, and the aa-9. A WET Labs Modular Ocean Data and Power System was used to power the instruments, acquire data, time stamp the data, and transmit the data up a single cable to an archiving Pentium processor PC. Simultaneous CTD measurements were necessary for temperature and salinity corrections applied to absorption coefficients during postprocessing [see section 2e(1)]. A prototype buoyancy controller was also on the profiler to control descent rate with respect to density (usually 3 to $10 \mathrm{~cm} \mathrm{~s}^{-1}$ ). The entire instrumentation package was designed to provide high-resolution, microscale $(\mathrm{cm})$ vertical profiles in coastal regions (Donaghay et al. 1992; Donaghay et al. 1996).

The aa-9 measured dual $a_{g}(\lambda)$. A variable speed gear pump at the surface was connected to the exiting " $\mathrm{Y}$ " of the aa-9 flow cells via $35 \mathrm{~m}$ of polyethylene tubing. For every cast, the flow rate through the aa- 9 was measured in the outflow of the surface pump in order to account for the time lag between a sample entering the filter and the sample measurement in the flow cells [see section $2 \mathrm{~d}(2)]$. Attached to the "Y" splitting the flow entering the aa- 9 was a length of Teflon tubing extending down to a 0.2- $\mu \mathrm{m}$ maxicapsule filter (Gelman) attached horizontally at the sampling depth. To decrease the resistance to flow, the outer capsule of the filter was removed, thereby preventing particle buildup on the filter surface. Increasing the flow rate provided better resolution of $a_{g}(\lambda)$ microstructure. Increased flow rates and more stable, cavitation-free measurements were also obtained by soaking a filter in Nanopure water for several hours before use. Filters could be used continuously for $2-4 \mathrm{~h}$ in these relatively productive coastal waters. Care was taken to ensure that the magnitude and changes with depth of $a_{g}(\lambda)$ were identical before and after a filter was replaced. Highest flow rates with a filter attached were $800-1200 \mathrm{~mL} \mathrm{~min}^{-1}$ before cavitation occurred, evidenced by spiking in the data stream from bubbles.

A Seabird $3000 \mathrm{rpm}$ submersible pump was placed after the exiting " $\mathrm{Y}$ " of the ac-9 flow cells for measurements of $a_{t}(\lambda)$. A degassing "Y" was additionally inserted in the ac-9 outflow to eliminate any trapped air when the package was submerged. Air escaped through a Teflon insert in the top of the inverted "Y." Flow rates through the meter were about $3 \mathrm{~L} \mathrm{~min}^{-1}$, measured by attaching a flow meter to the outflow tube of the pump when submerged. Based on lab results showing evidence of particle sorting through a "Y," we used separate intake tubes for each flow cell. All tubing directly con- nected to the ac-9 was wrapped in black vinyl tape to prevent light piping into the flow cells.

For some profiles, a second ac- 9 replaced the aa- 9 and $a_{t}(\lambda)$ was recorded in replicate. In these cases, the pump and plumbing configuration used for the first ac-9 was duplicated for the second ac-9.

\section{2) Time LagS}

Time lags were applied to profile data from the absorption meters to account for the time required for a water parcel to enter the intake tube and travel to the flow cell. Adjusting the time enabled the absorption profiles to be aligned with hydrographic parameters. For the ac-9s measuring $a_{t}$, the total volume of a flow cell and its associated plumbing was about $30 \mathrm{~mL}$. Since the flow rate was about $25 \mathrm{~mL} \mathrm{~s}^{-1}$ (split flow), the time lags were typically around $1.2 \mathrm{~s}$.

Time lags were more difficult to apply to the aa-9 data because the flushing behavior of the filter (complete mixing in the filter vs a "plug flow") and the functional volume of the filter were not known. As a result, time lags for the aa- 9 data were alternatively obtained by aligning consecutive in situ profiles of different descent rates but equal aa- 9 flow rates. Once the correct time lag was chosen, the profiles overlapped. Using this method, a time lag of $12 \mathrm{~s}$ was obtained for a flow rate of $1 \mathrm{~L} \mathrm{~min}{ }^{-1}$. Another technique used effectively by W. S. Pegau (1998, personal communication) is to align uncorrected absorption profiles at 715 or $750 \mathrm{~nm}$ with CTD temperature profiles. In cases where the temperature dependency of water absorption [see section 2e(1)] dominates the signal in the near-infrared, the profiles can be aligned.

\section{3) SPECTRAL SLOPE PARAMETER}

Any spectral analysis of $a_{g}$ requires a high level of accuracy in absorption measurements, partly because the natural variation in the spectral shape is relatively small. Many investigators have used an exponential curve fit to model CDOM absorption as a function of wavelength in the visible domain (e.g., Bricaud et al. 1981; Zepp and Schlotzhauer 1981; Carder et al. 1989):

$$
a_{g}\left(\lambda_{0}\right)=a_{g}\left(\lambda_{0}\right) e^{-s\left(\lambda-\lambda_{0}\right)}
$$

where $s$ is the spectral slope of the CDOM absorption spectrum when plotted on a natural logarithmic scale of absorption. Changes in $s$ have been associated with variations in the composition of the CDOM pool (Zepp and Schlotzhauer 1981; Carder et al. 1989).

Vertical profiles of $s$ were computed from exponential curve fits at a single depth through spectral $a_{g}(\lambda)$ profiles ( $\lambda$ range of 412 to $560 \mathrm{~nm}, n=5$ ). Before the calculation, $a_{g}$ for each wavelength was smoothed with a 20-point running mean (3.4-s bins). The calculation did not use $a_{g}(630), a_{g}(650)$, and $a_{g}(676)$ because the values in some cases were too low to provide consistent 
TABLE 1. Salinity corrections for absorption and attenuation for a 30 psu purified artificial seawater solution blanked against Nanopure water, and the resulting slopes for the ac-9 salinity correction. Slopes for the temperature correction are reprinted from Pegau et al. (1997) and their slopes for the salinity correction are provided for comparison.

\begin{tabular}{|c|c|c|c|c|c|}
\hline \multirow{2}{*}{$\begin{array}{c}\lambda \\
(\mathrm{nm})\end{array}$} & $a_{\text {salt, } 30 \text { psu }}$ & $c_{\text {salt, } 30 \text { psu }}$ & $\Psi_{s, a}$ & $\Psi_{s, c}$ & \multirow{2}{*}{$\begin{array}{c}\Psi_{t} \\
\left(\mathrm{~m}^{-1}{ }^{\circ} \mathrm{C}^{-1}\right)\end{array}$} \\
\hline & \multicolumn{2}{|c|}{$\left(\mathrm{m}^{-1}\right)$} & \multicolumn{2}{|c|}{$\left(\mathrm{m}^{-1} \mathrm{psu}^{-1}\right)$} & \\
\hline 412 & 0.0079 & 0.0020 & $0.00026 \quad(0.00018)^{*}$ & $0.00007 \quad(0.00007)^{*}$ & 0.0001 \\
\hline 440 & 0.0048 & -0.0013 & $0.00016 \quad(0.00008)$ & $-0.00004(-0.00007)$ & 0.0000 \\
\hline 488 & 0.0028 & -0.0023 & $0.00009 \quad(0.00008)$ & $-0.00008(-0.00007)$ & 0.0000 \\
\hline 532 & 0.0014 & -0.0039 & $0.00005 \quad(0.00004)$ & $-0.00013(-0.00008)$ & 0.0001 \\
\hline 555 & 0.0011 & -0.0031 & $0.00004 \quad(0.00008)$ & $-0.00010(-0.00008)$ & 0.0001 \\
\hline 630 & 0.0017 & -0.0046 & $0.00005 \quad(-)$ & $-0.00015 \quad(-)$ & 0.0002 \\
\hline 676 & 0.0005 & -0.0032 & $0.00002 \quad(0.00007)$ & $-0.00011(-0.00007)$ & 0.0001 \\
\hline 715 & -0.0063 & -0.0110 & $-0.00021(-0.00018)$ & $-0.00037(-0.00032)$ & 0.0029 \\
\hline 750 & 0.0167 & 0.0136 & $0.00056 \quad(0.00075)$ & $0.00045 \quad(0.00064)$ & 0.0106 \\
\hline
\end{tabular}

* values in parentheses are from Pegau et al. (1997).

curve fits. After the profile of spectral slope was obtained, the data was passed through a low-pass Butterworth filter (MATLAB) with a cutoff frequency of 0.2 Hz.

It is important to note that $s$ is particularly sensitive to inaccurate temperature, salinity, and water offset drift corrections. All the corrections have a wavelength dependence that affects the spectral properties of $a_{g}(\lambda)$. An example of this sensitivity is the application of an incorrect time lag to $a_{g}$ vertical profiles, where the temperature and salinity will be vertically offset with respect to $a_{g}$. Through a strong thermocline and/or halocline an artificial maximum or minimum in $s$ can occur within the gradient. Since time lags can be difficult to estimate when a sample passes through an inline filter [see section $2 \mathrm{~d}(2)$ ], care should be used in the interpretation of such features.

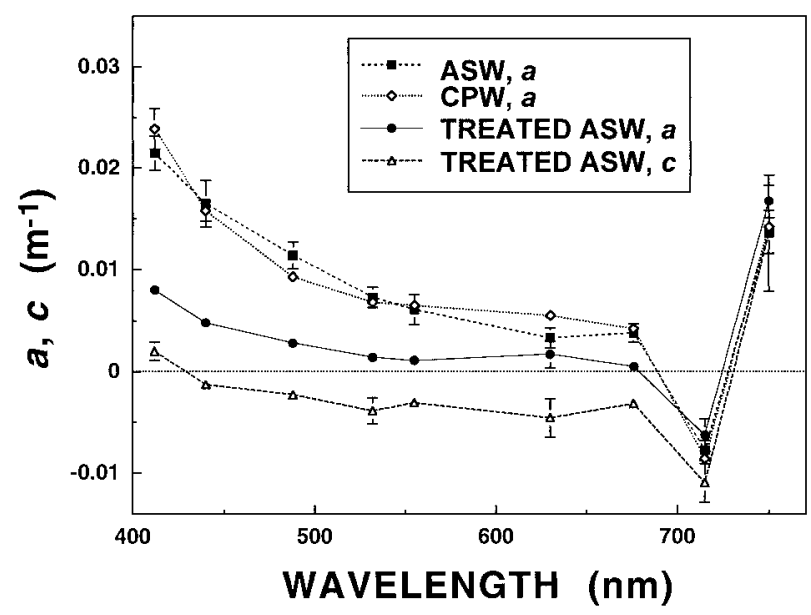

FIG. 3. Spectral absorption for artificial seawater (ASW), treated ASW, and a $0.2-\mu \mathrm{m}$ filtered sample collected from $15^{\circ} \mathrm{N}$ in the central Pacific (CPW). The ASW was a twice $0.2-\mu \mathrm{m}$ filtered, $30 \mathrm{ppt}$ solution of sea salts (Sigma) dissolved in Nanopure water. See text for a description of the purification treatments. The sample from the Pacific (36.5 ppt) was collected with trace metal clean techniques and $0.2-\mu \mathrm{m}$ filtered (provided courtesy of A. K. Hanson).

\section{e. Additional corrections}

\section{1) Temperature AND SALinity}

All absorption measurements and water calibrations were normalized to a constant temperature to account for the temperature dependence of the absorption coefficient of pure water (Pegau and Zaneveld 1993; Pegau and Zaneveld 1994; Pegau et al. 1997). Temperature effects are especially relevant at the harmonic frequencies of $-\mathrm{OH}$ bond stretching in the infrared and around $610 \mathrm{~nm}$ (Sullivan 1963). The correction was

$$
a_{\text {tcorr }}(\lambda)=a_{\text {meas }}(\lambda)-\left(T_{i}-T_{\text {norm }}\right) \Psi_{t}(\lambda),
$$

where $a_{\text {tcorr }}$ is the absorption corrected for changes in water temperature, $a_{\text {meas }}$ is the measured absorption at the in situ temperature, $T_{i}$ is the in situ temperature, $T_{\text {norm }}$ is the temperature that the absorption is being normalized to, and $\Psi_{t}$ is the wavelength specific slope, $\Delta a / \Delta T\left(\mathrm{~m}^{-1}{ }^{\circ} \mathrm{C}^{-1}\right)$. As reproduced in Table $1, \Psi_{t}(\lambda)$ from Pegau et al. (1997) are the most current published values and corroborate unpublished experimental work in our laboratory.

A salinity correction was also necessary due to the absorption by dissolved salts and refractive index errors between seawater and freshwater that can distort the optical path. Obtaining optically clean seawater for the correction is a difficult task, however, because some light-absorbing organic materials are very resistant to many purification techniques. Commercially obtained chemical grade sea salts or the individual salt fractions $\mathrm{NaCl}, \mathrm{MgCl}_{2}$, and $\mathrm{CaSO}_{4}$ are not free of organic contamination, and, thus, are not suitable salt blanks when dissolved in pure water. This contamination is large enough so that artificial seawater prepared with sea salts (Sigma) has an absorption spectrum nearly identical to that of a $0.2-\mu \mathrm{m}$ filtered sample collected from the central Pacific Ocean at $15^{\circ} \mathrm{N}$ (Fig. 3). If the spectrum from the artificial seawater was applied as a salinity blank to this oceanic sample, the resulting spectral CDOM absorption would be essentially zero.

We have determined salinity corrections for ac-9 ab- 
sorption and attenuation by purifying a 30 psu solution of sea salts dissolved in Nanopure water. The artificial seawater was subjected to a purification process consisting of activated charcoal treatments, artificial UV exposures, hydrogen peroxide ( $30 \%$ solution, no stabilizer) additions, and incubations in direct sunlight. The salt blank was exposed to UV radiation by continually passing the sample through a quartz coil surrounding a cylindrical mercury vapor lamp. All the tubing in the system was acid-cleaned Teflon, and nitrogen pressure was used to force the sample through the coil, avoiding any contamination from an inline pump. For all measurements, the salts solution was $0.2-\mu \mathrm{m}$ filtered before entering the ac-9.

The treatments were continued until no detectable changes in the absorption or attenuation of the salt blank were observed from continuous UV exposure. The resulting values for the salinity correction in the visible and near-infrared are provided in Table 1 and plotted in Fig. 3. From 412 to $676 \mathrm{~nm}$, the salt corrections in the final sample were the lowest values measured. However, these values are used as upper limits for the salt correction, acknowledging the possibility that some lightabsorbing organic material resistant to the above purification methods may have remained in solution. Salt corrections at 715 and $750 \mathrm{~nm}$ were also taken from measurements made in the final salt blank sample, although these values showed little variation through the purification process because of the very low absorption signal from organic material in the infrared. Since preliminary results from our lab and the work of Pegau et al. (1997) indicate that the corrections over varying concentrations of dissolved salt are linear with increasing salinity, slopes of the salt corrections $\left(\mathrm{m}^{-1} \mathrm{psu}^{-1}\right)$ are also given in Table 1. The slopes compared well with those of Pegau et al. (1997). In the visible, the largest differences were at 412 (44\% higher) and $440 \mathrm{~nm}(50 \%$ higher), possibly the result of residual organics in our sea salts solution or from slight differences in the optics of the ac-9s used in each study.

Salinity corrections using these slopes were applied to absorption and attenuation data as follows:

$$
\begin{aligned}
& a_{\text {scorr }}(\lambda)=a_{\text {meas }}(\lambda)-S_{i} \Psi_{s, a}(\lambda), \\
& c_{\text {scorr }}(\lambda)=c_{\text {meas }}(\lambda)-S_{i} \Psi_{s, c}(\lambda),
\end{aligned}
$$

where $a_{\text {scorr }}$ and $c_{\text {scorr }}$ are the absorption and attenuation, respectively, corrected for all salinity effects (normalized to $S=0 \mathrm{psu}$ ), $S_{i}$ is the salinity of the sample, $a_{\text {meas }}$ and $c_{\text {meas }}$ are the measured absorption and attenuation at salinity $S_{i}, \Psi_{s, a}$ is the wavelength specific slope for absorption, $\Delta a / \Delta S\left(\mathrm{~m}^{-1} \mathrm{psu}^{-1}\right)$, and $\Psi_{s, c}$ is the wavelengthspecific slope for attenuation, $\Delta c / \Delta S\left(\mathrm{~m}^{-1} \mathrm{psu}^{-1}\right)$.

\section{2) SCATTERING ERROR}

When measuring absorption in a particle field with an ac-9, a scattering correction is necessary to estimate the portion of lost light scattered at angles greater than $41^{\circ}$, the angle of total internal reflection (Zaneveld et al. 1994). This scattered light usually accounts for only $8 \%-15 \%$ of the total scattering because of the reflective quartz design of the flow cell with diffuser at the collection end (Zaneveld and Bartz 1984; Zaneveld et al. 1994). Scattering corrections were applied to temperature, salinity, and drift-corrected total absorption measurements by subtracting corrected values at $750 \mathrm{~nm}$ from the absorption measured by all other wavelengths. This is one of the methods proposed by Zaneveld et al. (1994) for correcting the scattering error in the ac-9. The primary assumptions are 1) there is minimal absorption at $750 \mathrm{~nm}$, so the measured values are only from the scattering error, and that 2) the scattering error is independent of wavelength.

A scattering correction that incorporates measurements of the total scattering spectrum (the "proportional correction") has been demonstrated to be more accurate than the constant correction method (Zaneveld et al. 1994). We have found it difficult to apply, however, because of the high-frequency variability in $a_{t}(\lambda)$ and $c_{t}(\lambda)$ measurements due to particles. This variability is coupled between any of the wavelengths in one optical path but is uncoupled between different optical paths. One option is to low-pass filter the data from either or both paths and then apply the proportional correction. However, this is done at the risk of removing potentially real microscale variability from the absorption measurements. A further analysis of the effects of applying the proportional correction is currently in progress.

\section{3) SUMMARY OF CORRECTIONS}

Final, corrected CDOM absorption coefficients, $a_{g}(\lambda)$, collected with a $0.2-\mu \mathrm{m}$ prefilter, were obtained from raw values, $a_{\text {meas }}(\lambda)$, using

$$
\begin{aligned}
a_{g}(\lambda)= & a_{\text {meas }}(\lambda)-D(\lambda)-\left(T_{i}-T_{\text {norm }}\right) \Psi_{t}(\lambda) \\
& -S_{i} \Psi_{s, a}(\lambda)
\end{aligned}
$$

and final, corrected total absorption coefficients, $a_{t}(\lambda)$, were obtained from raw values using

$$
\begin{aligned}
a_{t}(\lambda)= & a_{\text {meas }}(\lambda)-D(\lambda)-\left(T_{i}-T_{\text {norm }}\right) \Psi_{t}(\lambda) \\
& -S_{i} \Psi_{s, a}(\lambda)-a_{t}(750),
\end{aligned}
$$

where $a_{t}(750)=a_{\text {meas }}(750)-D(750)-\left(T_{i}-T_{\text {norm }}\right)$ - $\Psi_{t}(750)-S_{i} \Psi_{s, a}(750)$. In all cases, the absorption of pure water was subtracted in $a_{\text {meas }}$ in data acquisition. The $a_{t}(\lambda)$ and $a_{g}(\lambda)$ are corrected for temperature, salinity, and instrument drift, and $a_{t}(\lambda)$ is additionally corrected for scattering. The term $D(\lambda)$ is the drift correction obtained from the water calibrations.

Particulate absorption $a_{p}(\lambda)$ was then computed from

$$
a_{p}(\lambda)=a_{t}(\lambda)-a_{g}(\lambda) .
$$

\section{The ac-9 long-term drift}

Calibration records spanning 183 days were compiled for one ac-9, ac90142, as an example of long-term drift 

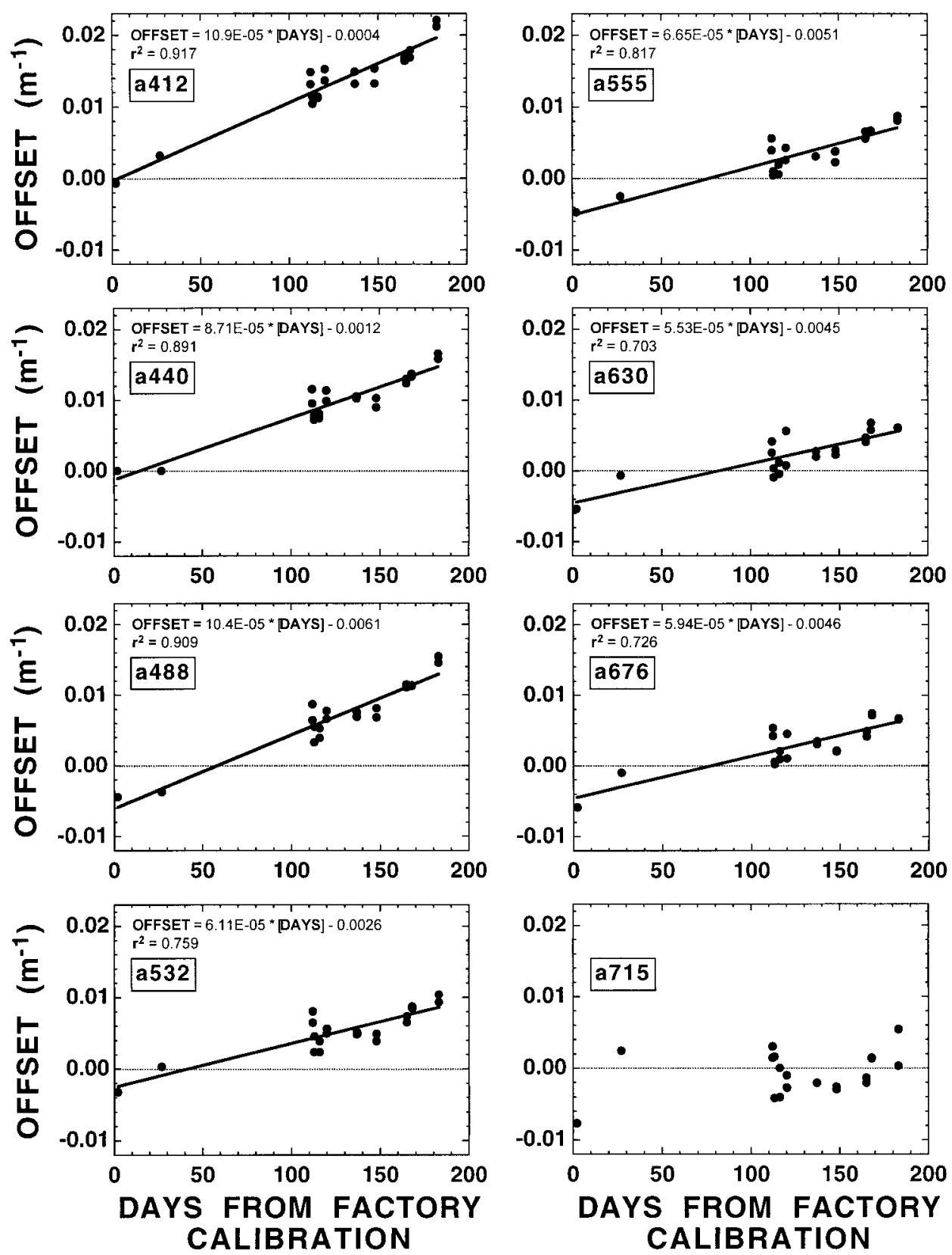

FIG. 4. Water offset drifts for the absorption and attenuation channels of ac 90142 based on laboratory water calibrations after the WET Labs calibration 21 Aug 1995. All the drifts have been temperature corrected. The wavelength, linear regression, and error coefficient are displayed in the graphs as appropriate.

in a relatively stable meter (Fig. 4). The instrument was originally calibrated by WET Labs with pure water (21 August 1995). Using Eq. (3), all measurements were normalized to the temperature of the original WET Labs calibration, $24.3^{\circ} \mathrm{C}$, for intercomparison.

Water offset drifts in ac90142 were best approximated by a linear least squares regression, which was applied to the absorption and attenuation channels (Fig. 4). The rates of drift increased with decreasing wavelength in both the absorption and attenuation channels. No re- gression is given for slopes not significantly different from zero ( $t$ test, $p>0.05$ ). The drifts may be due in part to partial degradation of the interference filters, where filters used for shorter wavelength, higher energy radiation drift more rapidly (D. Hankins, WET Labs, 1995, personal communication). The highest rates of drift in both absorption and attenuation channels were about $0.0001 \mathrm{~m}^{-1} \mathrm{day}^{-1}$ at $412 \mathrm{~nm}$.

Between replicate calibrations the agreement at any wavelength was always better than $0.005 \mathrm{~m}^{-1}$, and usu- 

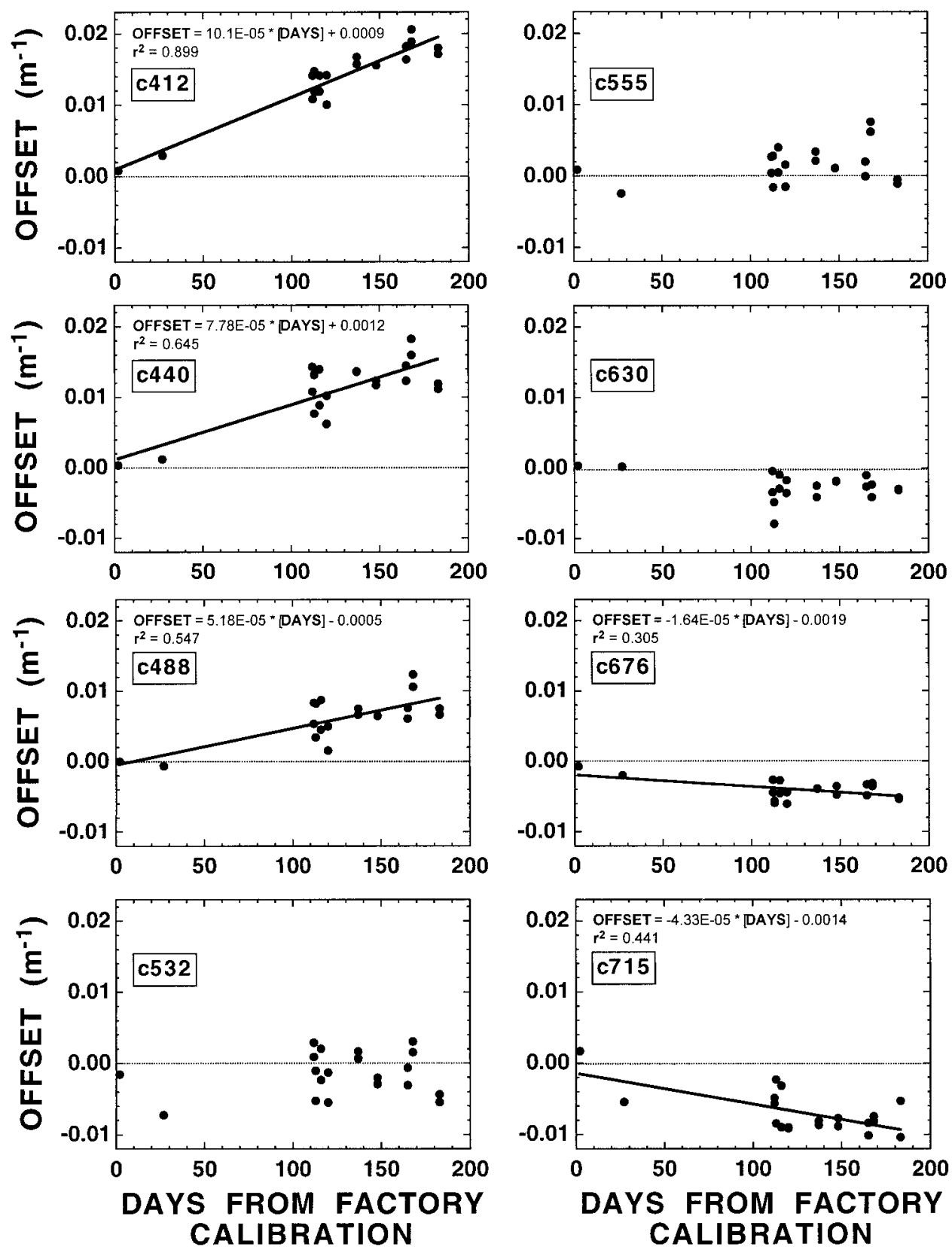

FIG. 4. (Continued)

ally better than $0.002 \mathrm{~m}^{-1}$. To correct spectral absorption measurements on any calibration day, a mean water offset drift was obtained and subtracted from measured values. In some cases, drifts were calculated for days between calibrations by linear interpolation. The precision of the drift correction was normally better than $\pm 0.003 \mathrm{~m}^{-1}$.

The drifts obtained from both air and water calibrations were periodically measured for another meter, ac90143, to determine the effectiveness of using air calibration drifts to correct for water offset drifts. After 88 days, there was a significant residual between absorption channel offsets derived from water and those from air calibrations that was wavelength dependent (Table 2). Since drifts determined by air and water calibrations were not equivalent, instrument drift could not be corrected using air calibrations alone. The residuals probably arise from small changes in the optical surfaces of the pressure windows (microscopic scratches from periodic cleaning or persistent organic material) that alter the reflectivity of the air/quartz and water/quartz interfaces by different amounts. 
TABLE 2. Residuals between calibrations with dry $\mathrm{N}_{2}$ gas ("air") and pure water 88 days after the calibrations by WET Labs. Offset drifts, $D_{a}$ and $D_{w}$, are for air and water calibrated, respectively.

\begin{tabular}{cc}
\hline \hline$\lambda(\mathrm{nm})$ & $D_{a}-D_{w}\left(\mathrm{~m}^{-1}\right)$ \\
\hline 412 & 0.0114 \\
440 & 0.0089 \\
488 & 0.0057 \\
520 & 0.0057 \\
560 & 0.0033 \\
630 & 0.0049 \\
676 & 0.0044 \\
715 & 0.0040 \\
\hline
\end{tabular}

\section{Correcting in situ measurements}

Data from three vertical profiles collected on 15 June 1995, 31 May 1996, and 29 August 1996 in East Sound, Washington, will be used to evaluate the effects of water calibration, temperature, and salinity corrections on absorption data in situ. In June 1995 and May 1996, aa90106 was used to measure $a_{g}(\lambda)$ in replicate. Both ac90143 and ac90142 were used to additionally record total absorption on the June 1995 and May 1996 cruises, respectively. The August 1996 profile will be used to compare total absorption coefficients collected using two different meters, ac 90142 and ac90143. Temperature and salinity data for each profile are plotted in Fig. 5.

\section{a. The $a_{g}$ vertical profiles}

To accurately assess the effectiveness of the corrections, $a_{g}(\lambda)$ vertical profiles can be more useful than $a_{t}(\lambda)$ because the high-frequency variability in $a_{g}(\lambda)$ measurements is usually much lower than the variability in a particle field. The profile from 31 May 1996 will be used for the exercise. The two absorption channels at $412 \mathrm{~nm}$ were separated by a constant $0.036 \mathrm{~m}^{-1}$ offset in the untreated, raw data (Fig. 6a). After the temperature and salinity corrections, the profiles were shifted lower by 0.003 to $0.008 \mathrm{~m}^{-1}$ (Fig. 6b). Additionally applying the correction for water offset drift aligned the profiles within $0.005 \mathrm{~m}^{-1}$ (Fig. 6c). The water offset drift corrections were up to an order of magnitude greater than other corrections. In the same cast, the $a_{g}(560)$ channels were separated by a $0.037 \mathrm{~m}^{-1}$ offset, and the $a_{g}(676)$ channels were $0.038 \mathrm{~m}^{-1}$ apart at the surface (Figs. 7a,b). After the corrections, the two $a_{g}(560)$ channels were aligned within $0.003 \mathrm{~m}^{-1}$ (Figs. 7c,d). The two $a_{g}(676)$ channels overlapped at the bottom but were separated by an offset of about $0.008 \mathrm{~m}^{-1}$ at the surface. Inconsistent offsets with depth appear to be instrument and wavelength specific. The exact cause is currently unclear.

Therefore, although the precision in the raw absorption values were $\pm 0.001 \mathrm{~m}^{-1}$ or less, the "operational" precision, defined as the reproducibility between different absorption channels used simultaneously, is generally \pm 0.002 to $\pm 0.005 \mathrm{~m}^{-1}$ depending on the meter(s) used and the wavelength tracked. Furthermore, the water offset drift corrections obtained from calibrations become increasingly important at longer wavelengths where, in many cases, natural CDOM absorptions are lower than the magnitude of the correction.

\section{$b$. The $a_{t}$ vertical profiles}

Two simultaneously recording ac-9s were used to determine the effects of the temperature, salinity, calibration, and scattering corrections on profiles of total ab-

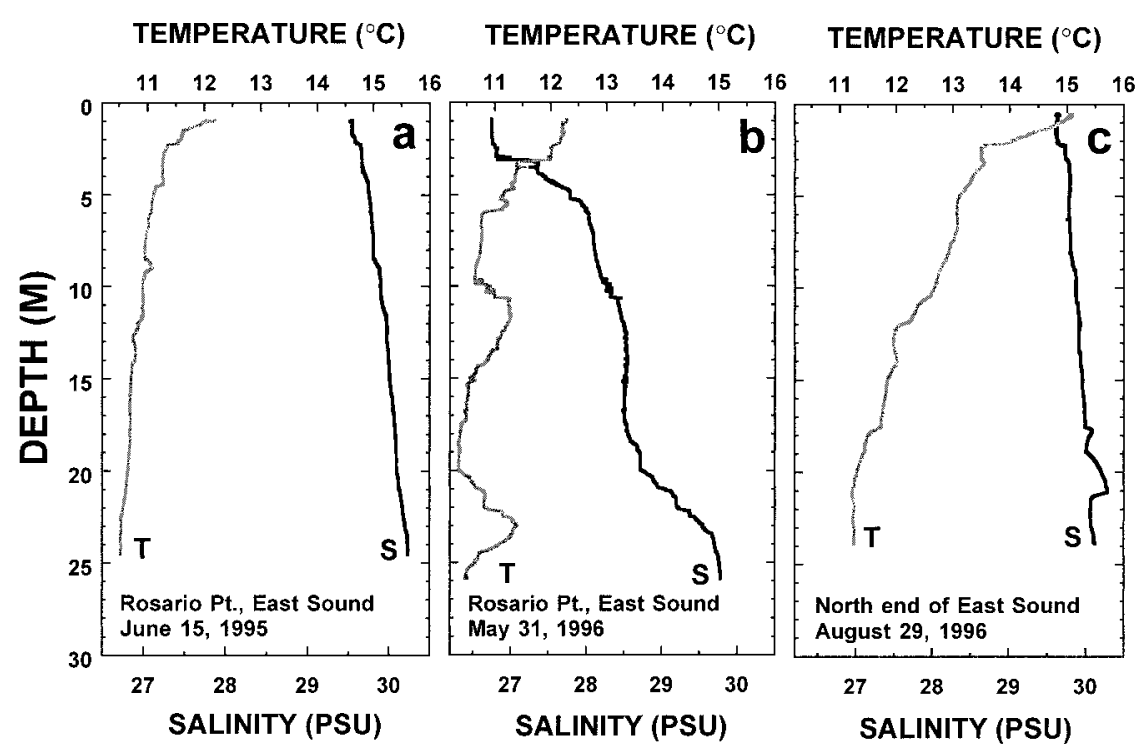

FIG. 5. Temperature and salinity from three vertical profiles collected on (a) 15 Jun 1995, (b) 31 May 1996, and (c) 29 Aug 1996 in East Sound, WA. 


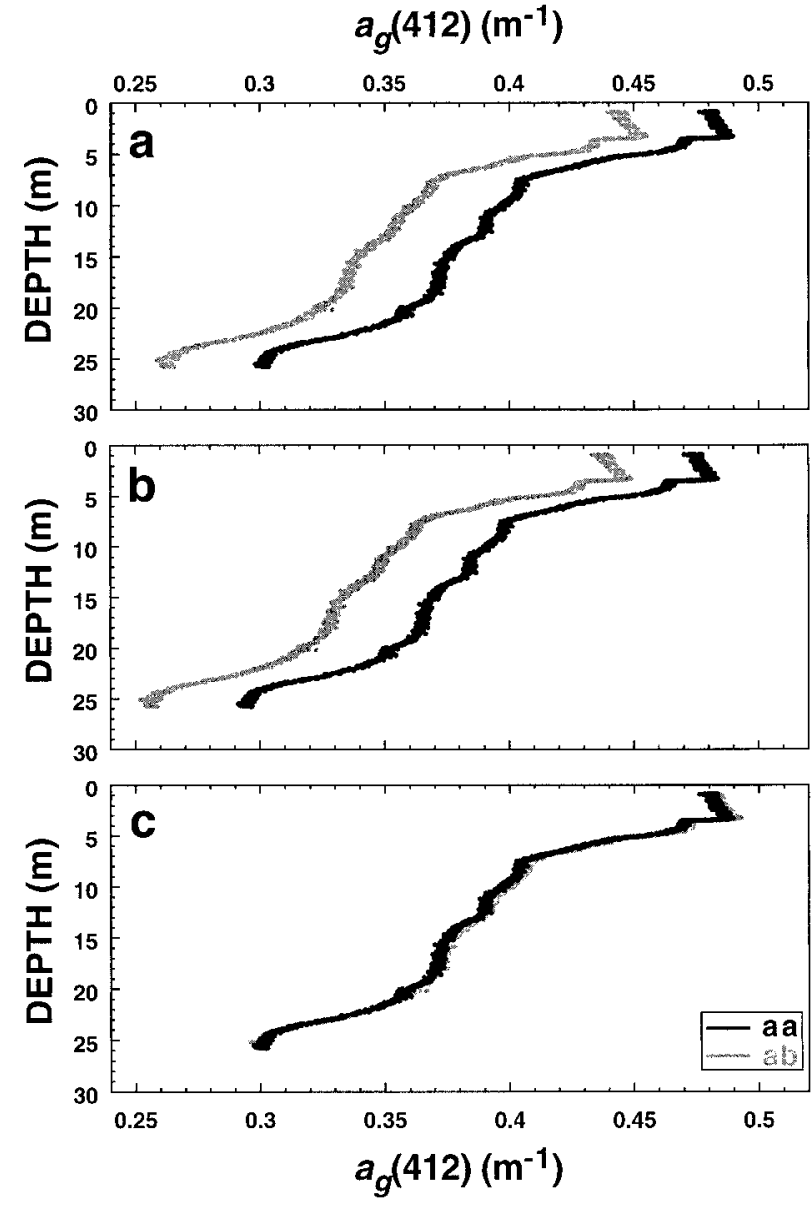

FIG. 6. Vertical profiles $a_{g}(412)$ measured in replicate with the aa90106 on 31 May 1996. (a) The untreated, raw profiles measured by each channel; (b) the profiles after temperature and salinity corrections were applied; and (c) the profiles after the calibration corrections were additionally applied.

sorption and the degree of replication between different meters. Total absorption coefficients at 440 and $676 \mathrm{~nm}$ are plotted due to their importance in estimating phytoplankton pigment absorption. In the East Sound profile from 29 August 1996, the offsets between the two meters at $a_{t}(440)$ and $a_{t}(676)$ were about 0.10 and 0.08 $\mathrm{m}^{-1}$, respectively (Figs. 8a,b). A broad absorption maximum was present between 4 and $10 \mathrm{~m}$. The offsets appeared consistent through the water column when the data from one meter was directly plotted against data from the other (Figs. 9a,b; gray circles). The least squares linear regression for $a_{t}(440)$ was between 0.09 and $0.10 \mathrm{~m}^{-1}$ from the $1: 1$ line, representing a zero offset between the meters, and the regression for $a_{t}(676)$ was between 0.07 and $0.09 \mathrm{~m}^{-1}$ from the 1:1 line.

After applying the temperature, salinity, water offset drift, and scattering corrections to the profiles, the offsets between the two meters for both $a_{t}(440)$ and $a_{t}(676)$ were significantly reduced (Figs. 8c,d and 9a,b; black dots). The ac90142 water offset drift corrections were
0.0260 and $0.0104 \mathrm{~m}^{-1}$ for $a_{t}(440)$ and $a_{t}(676)$, respectively. The ac 90143 water offset drift corrections were -0.0472 and $-0.0482 \mathrm{~m}^{-1}$ for $a_{t}(440)$ and $a_{t}(676)$, respectively. Small offsets were still present after the corrections, however, with the linear regression through the corrected data deviating from the 1:1 zero offset line anywhere from 0.003 to $0.02 \mathrm{~m}^{-1}$ in $a_{t}(440)$ and 0.004 to $0.02 \mathrm{~m}^{-1}$ in $a_{t}(676)$ (Figs. 9a,b). When considering that the precision in the measurement of total absorption, even in a relatively dilute particle field, is usually greater than $\pm 0.03 \mathrm{~m}^{-1}$, the residual offsets in the regressions were considered minor.

The variability in total absorption between the two meters appears to be due to relatively large particles, such as marine snow, in a patchy microscale distribution, creating a heterogeneous absorption field. The average total absorption from each meter is approximately equal, but the correlation of the high-frequency variability is poor from each meter sampling different microscale patches at slightly different times. Further study is required to characterize the exact source of the "noise," and the information it may contain about the distribution of particles on small scales.

\section{c. The $a_{t}, a_{g}$, and $a_{p}$ interrelationships}

The relationship between CDOM, total, and particulate absorption was explored to determine the relative contributions of the individual component absorptions to total absorption as a function of depth. It is important that the measured IOPs are consistent with theory (e.g., $a_{g}$ can never be greater than $a_{t}$ ) and realistic in relative magnitude. Additionally, the importance of measuring these IOPs on the microscale could be evaluated. For the following plots, all three parameters are plotted on the same scale for intercomparison.

In the June 1995 profile, total absorption at $440 \mathrm{~nm}$, $a_{t}(440)$, peaked at the surface at $0.58 \mathrm{~m}^{-1}$ but rapidly decreased to about $0.33 \mathrm{~m}^{-1}$ below $2 \mathrm{~m}$ (Fig. 10a). Above this steep gradient [43\% loss in $a_{t}(440)$ in about 2 -cm depth], $a_{p}(440)$ was $0.41 \mathrm{~m}^{-1}$, or $70 \%$ of $a_{t}(440)$ on average. Below the gradient, from 2-10 $\mathrm{m}$, the $a_{p}(440)$ and $a_{g}(440)$ contributions to the total were approximately equal.

A sharp, thin-layer particle peak centered around 3 m was evident in the May 1996 profile (Fig. 10b; see inlay for blowup). This peak structure is representative of persistent, thin-layered, phytoplankton patches that have been observed in East Sound and coastal shelf regions (Cowles and Desiderio 1993; Donaghay et al. 1996). The peak in $a_{p}(440)$ within the layer, $1.47 \mathrm{~m}^{-1}$, was approximately five times the $a_{p}(440)$ values $1 \mathrm{~m}$ above the peak and $1 \mathrm{~m}$ below the peak. Note that $a_{p}(440)$ accounted for $84 \%$ of $a_{t}(440)$ within the peak and about half the total just outside the peak. Throughout the rest of the water column, however, $a_{g}(440)$ was greater than $a_{p}(440)$ with the exception of a particle submaximum centered around $12 \mathrm{~m}$. Outside of the 


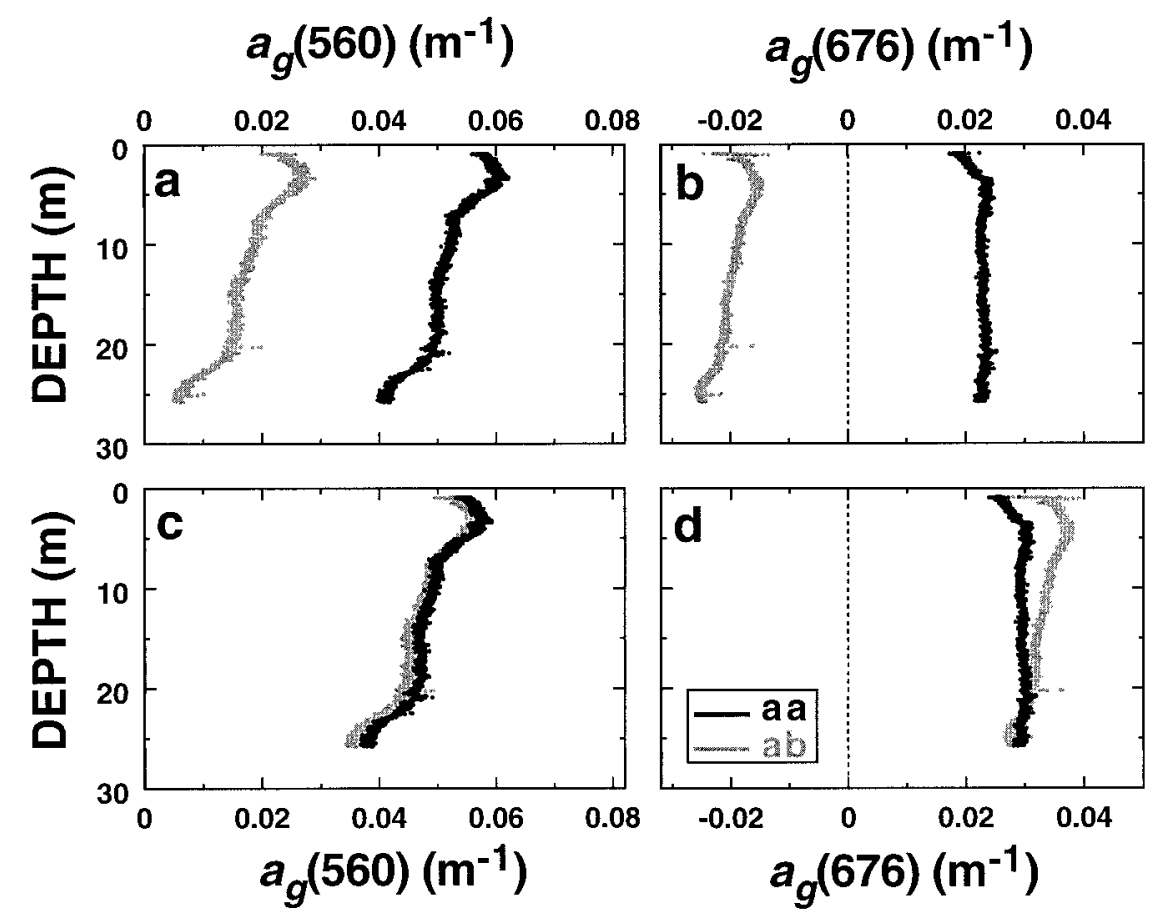

FIG. 7. Vertical profiles of $a_{g}(560)$ and $a_{g}(676)$ measured in replicate with the aa90106 on 31 May 1996. (a) and (b) The untreated, raw profiles measured by each channel; and (c) and (d) the profiles after the temperature, salinity, and calibration corrections were applied.

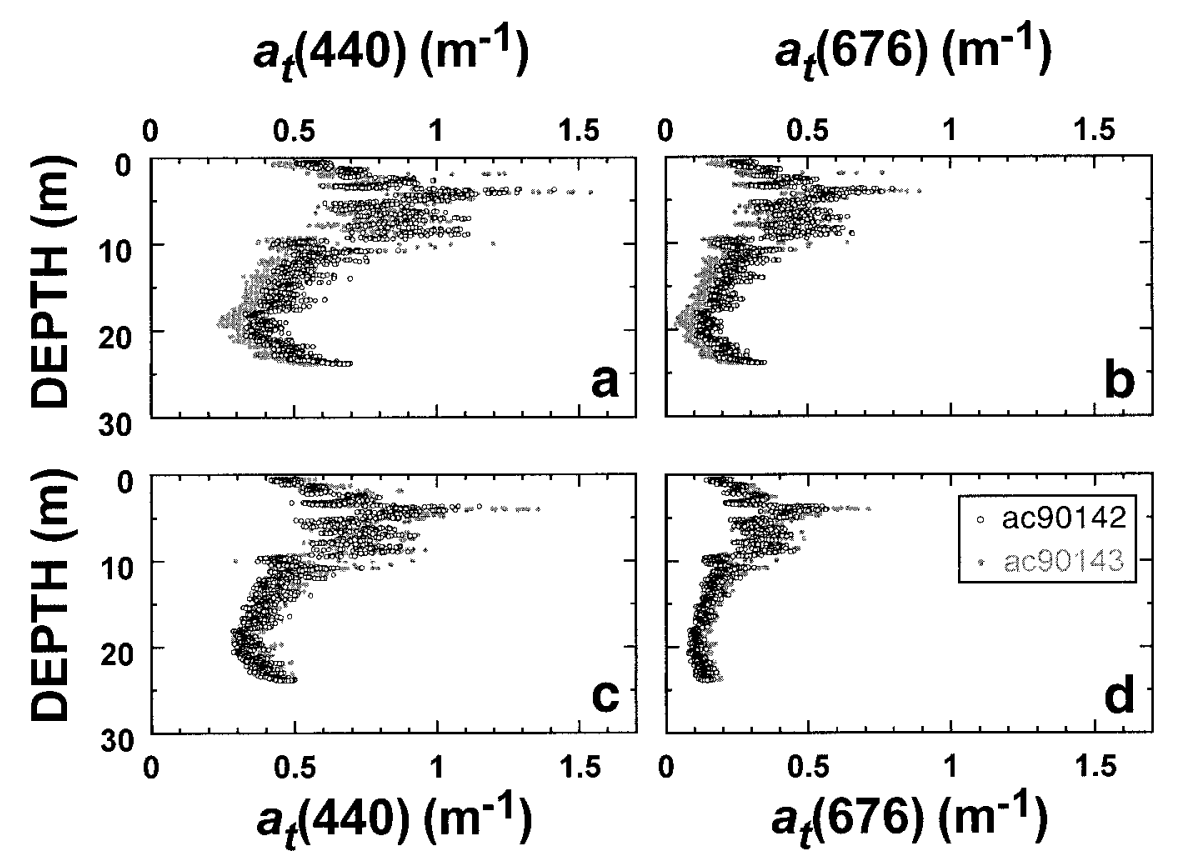

FIG. 8. Vertical profiles of $a_{t}(440)$ and $a_{t}(676)$ measured with ac90142 and ac90143, simultaneously. (a) and (b) are the untreated, raw profiles, and (c) and (d) are the profiles after temperature, salinity, scattering, and calibration corrections were applied. 

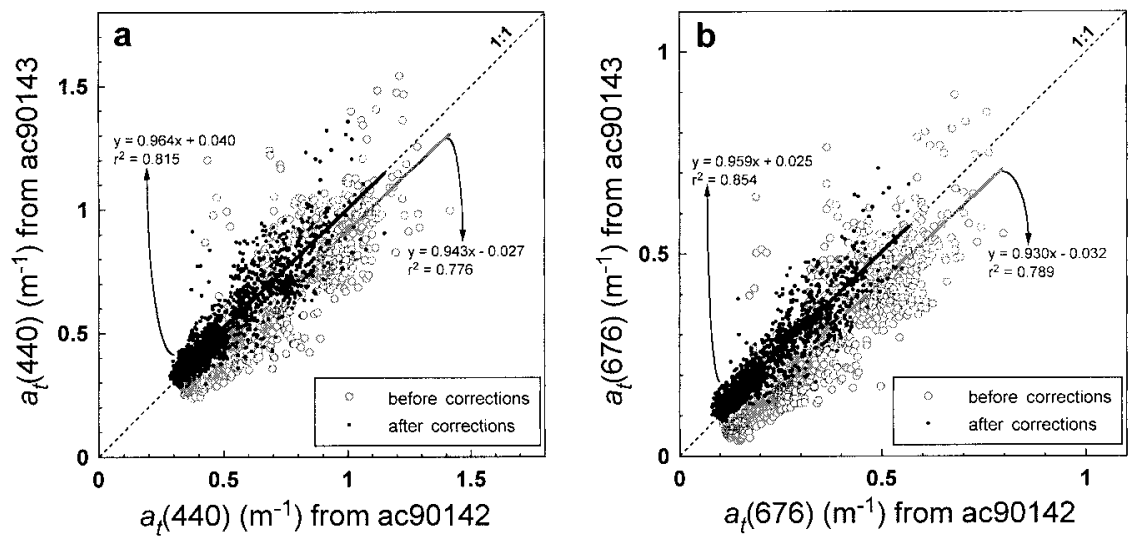

FIG. 9. The profiles of (a) $a_{t}(440)$ and (b) $a_{t}(676)$ from Fig. 8 with ac90142 plotted against ac90143. The gray, open circles with the solid gray regressions are the untreated, raw data, and the black circles with the solid black linear regression are the temperature, salinity, scattering, and calibration corrected data.

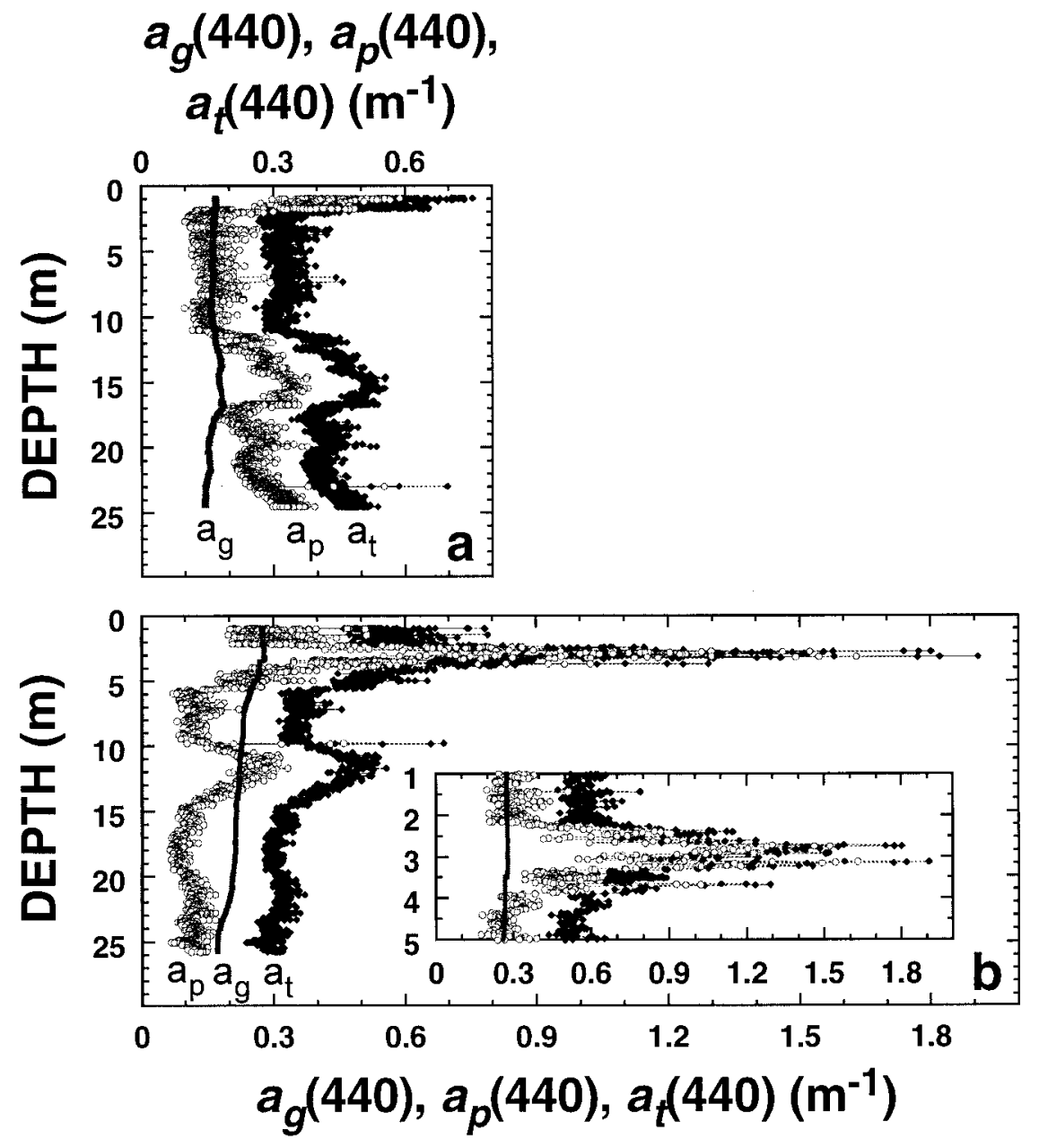

FIG. 10. Vertical profiles of $a_{g}(440), a_{t}(440)$, and $a_{p}(440)$ collected (a) 15 Jun 1995, and (b) 31 May 1996. A blowup of the 1- to 5-m region for 31 May 1996 is provided as an inlay in (b). 


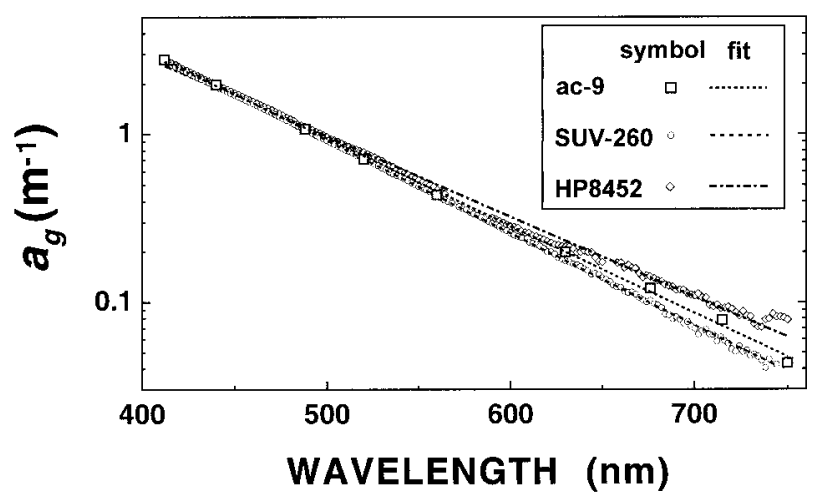

FIG. 11. Spectral $a_{g}$ of diluted coffee extract (5 ppt) as measured by the ac-9, the HP8452, and the SUV-260. HP8452 values from 570 to $590 \mathrm{~nm}$ are omitted due to faulty diodes. Each spectrum has been fit by an exponential curve. The exponential spectral slope was $\approx 0.0125 \mathrm{~nm}^{-1}$.

peak, the contribution of $a_{g}(440)$ to the total varied between $60 \%$ and $70 \%$. There were marked changes over depth intervals of less than a meter for $a_{g}, a_{p}$, and the contributions of $a_{g}$ and $a_{p}$ to $a_{t}$ in both profiles.

\section{Spectral relationships}

a. Spectral $a_{g}(\lambda)$

Corrected $a_{g}(\lambda)$ spectra of 5 ppt diluted coffee measured by the HP8452, the SUV-260, and the ac-9 were similar (Fig. 11). At all wavelengths, $a_{g}(\lambda)$ from the HP8452 was higher than $a_{g}(\lambda)$ from the SUV-260 by $0.03-0.10 \mathrm{~m}^{-1}$. Values from the ac-9 were between the two spectrophotometer readings, and, overall, compared better with the SUV-260 spectrum. The slightly higher values associated with the ac-9 relative to the SUV-260 may, in part, be due to the wider 10-nm bandwidth of the interference filters of the ac-9. Depending on the absorption spectrum of the sample and the spectral output of the lamp within the filter bandpass, widening the bandpass can alter (potentially up or down) estimates of absorption (R. Desiderio 1998, personal communication). The highest offset, $0.05 \mathrm{~m}^{-1}$, was recorded at the shortest wavelength, $412 \mathrm{~nm}$.

The coefficient $a_{g}(\lambda)$ was also determined in unialgal 8-L cultures of the diatom Asterionellopsis sp. in stationary growth phase. The resulting spectrum (Fig. 12) was described better by a power curve than the more commonly applied exponential decrease. Curves steeper than an exponential have been previously interpreted as evidence of a scattering error, even in cases in which a sample was filtered through a $0.2-\mu \mathrm{m}$ Nuclepore filter (Davies-Colley and Vant 1987). In this spectrum, the steeper curve was not the result of a scattering error from particulate contamination because 1) the absorption of Nanopure water with and without having passed it through the nylon capsule filter was measured and no contamination from fibers leaching into the filtrate was

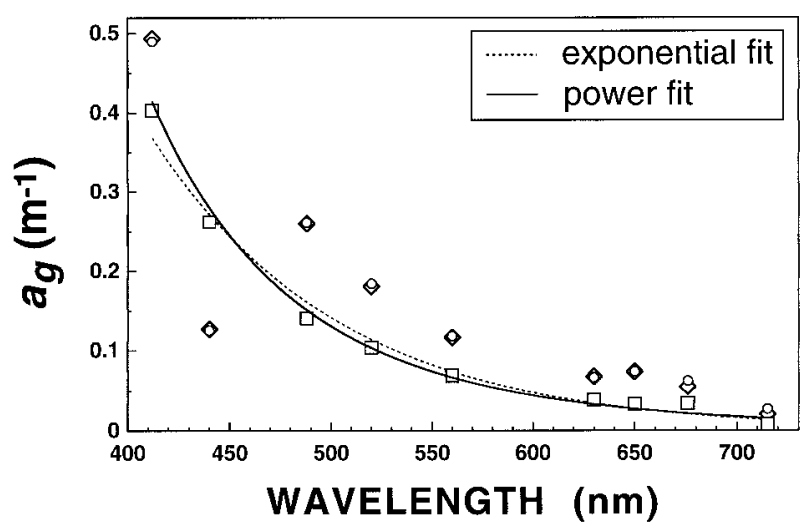

FIG. 12. Spectral $a_{g}$ from a culture of Asterionellopsis sp. measured with the ac-9. Diamonds are the raw values, gray circles are after temperature and salinity corrections, and the boxes are after an additional correction for calibrations. Both an exponential and power curve fit are provided.

detected, 2) a sample from a culture was filtered multiple times with the same result, and 3) simultaneous attenuation channel measurements were made that would clearly have indicated any particle contamination. CDOM absorption spectra in the visible that are steeper than an exponential may therefore be common in aquatic systems. Accumulating measurements of $a_{g}(\lambda)$ from ac-9s (Twardowski 1998) and the data of Carder et al. (1989) support this hypothesis. Although it is clear that the exponential spectral slope parameter still can be useful in determining spectral relationships, there is potentially more spectral information that should be investigated further.

In the May 1996 vertical profile, $s$ values were obtained from the two absorption channels of aa90106. The two profiles were similar in magnitude and shape through the water column (Fig. 13). Values ranging from 0.0138 to $0.0146 \mathrm{~nm}^{-1}$ are in agreement with previously published work in the visible domain (Bricaud et al. 1981; Zepp and Schlotzhauer 1981). In discrete samples from a wide variety of marine source regions, Zepp and Schlotzhauer (1981) and Bricaud et al. (1981) found variations of $0.0140 \pm 001$ and $0.0140 \pm 0032 \mathrm{~nm}^{-1}$, respectively. Overall, the vertical structure of $s$ in this profile appeared to mirror the temperature profile closely (Fig. 13). To eliminate the possibility of the relationship being an artifact in the temperature correction of absorption, a sensitivity analysis was performed on the spectral slope profiles. Spectral slopes were recalculated after the temperature correction was first omitted and then after it was doubled, and the results were compared to the original, corrected profile. Shifts in $s$ values from the sensitivity tests were less than $0.0001 \mathrm{~nm}^{-1}$. These adjustments did not affect the relationship between temperature and $s$.

\section{b. Spectral $a_{p}(\lambda)$}

Particulate absorption spectra were collected in the laboratory and in the field for intercomparison. During 


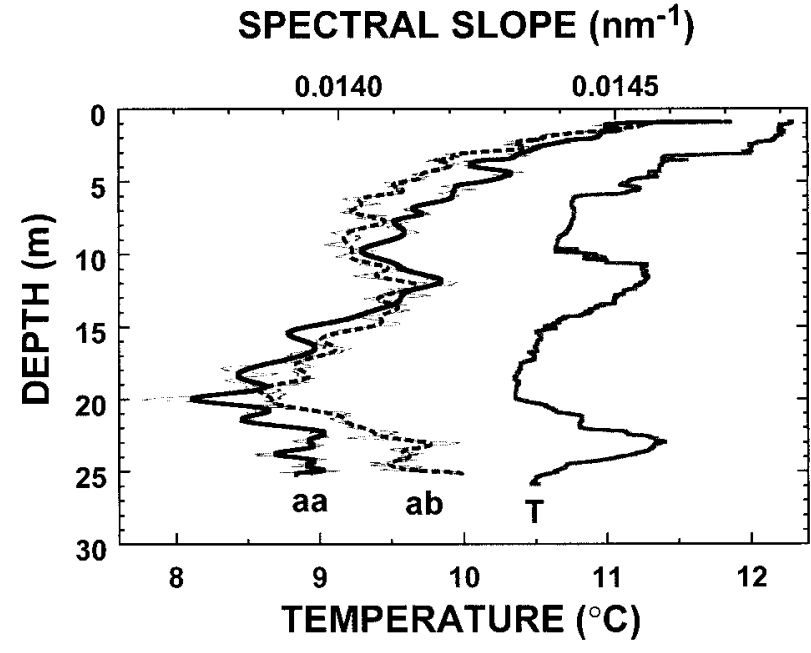

FIG. 13. Vertical profiles of spectral slope for the aa and ab channels of aa90106 from 31 May 1996. The solid and dashed lines are the spectral slopes after a low-pass filter, and the hairline lines are the original calculated $s$ values. The shape of the $s$ profiles compared well with temperature.

one occasion on 2 September 1996 in the surface water of East Sound, greater than $90 \%$ of the biomass of the phytoplankton fraction greater than $20 \mu \mathrm{m}$ was dominated by the diatoms Chaetoceros eibenii and Chaetoceros socialis (Rines, Sullivan, and Donaghay, unpublished data). At $2.8 \mathrm{~m}, a_{p}(\lambda), a_{t}(\lambda)$, and $a_{g}(\lambda)$ spectra were obtained from a vertical profile though the Chaetoceros patch (Fig. 14b). Peaks from chlorophyll absorption were evident at 440 and $676 \mathrm{~nm}$. Here $a_{p}$ was greater than $a_{g}$ at every wavelength except $412 \mathrm{~nm}$.
In Fig. 14a, $a_{p}(\lambda), a_{t}(\lambda)$, and $a_{g}(\lambda)$ spectra were also measured in a culture of Chaetoceros sp. In the culture $a_{p}(\lambda)$ was about four times higher than it was in the in situ measurement, and $a_{p}(\lambda)$ was a much larger proportion of $a_{t}(\lambda)$. To more closely compare the spectra, $a_{p}(\lambda)$ from the culture and the in situ measurement were normalized to $440 \mathrm{~nm}$ (Fig. 14c). A 440-nm normalized absorption spectrum of Chaetoceros sp. from Yentsch (1962) measured using the filter pad technique has also been overlaid for comparison. The three normalized spectra were similar, with the same general shape through the midvisible region and the strong peak at $676 \mathrm{~nm}$. The largest difference between the spectra was in the magnitude of the 676-nm chlorophyll peak, where the 440-nm normalized absorption at $676 \mathrm{~nm}$ was $25 \%$ lower in the culture than in either the in situ sample or the spectrum from Yentsch (1962). These spectral differences are within the range of normal physiological adaptations (e.g., package effects, varying concentrations of accessory pigments) from changing environmental conditions (Bricaud et al. 1995).

\section{Discussion}

\section{a. Instrument drift}

It is clear that several corrections must be applied to raw absorption data collected with the ac-9 to obtain accurate results. Water calibrations to correct for pure water offset drifts are an essential part of the methodology. For ac90142, the apparently low rate of drift of $0.0001 \mathrm{~m}^{-1}$ day $^{-1}$ at $412 \mathrm{~nm}$ does not imply that ac-9 water calibrations are unnecessary. Significant changes
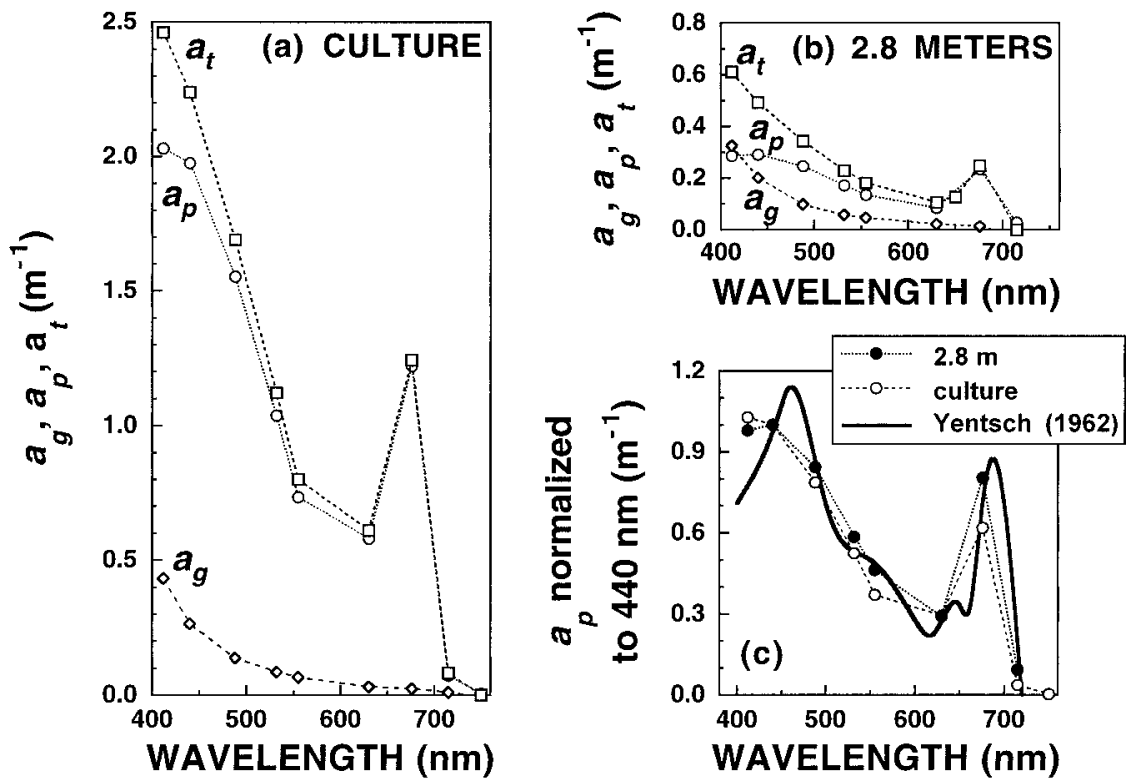

FIG. 14. Spectral $a_{t}, a_{p}$, and $a_{s}$ from (a) a laboratory culture of Chaetoceros vanheurckii, and (b) extracted from a vertical profile through a dense patch of Chaetoceros eibenii. Here, $a_{p}(\lambda)$ normalized to $a_{p}(440)$ for each spectrum are plotted in (c) with an overlay of 440 -nm normalized spectral attenuance of Chaetoceros sp. from Yentsch (1962) measured with the filter pad technique. 
in water offsets $\left(>0.01 \mathrm{~m}^{-1}\right)$ have occurred after an instrument has been physically stressed from shipping or deployment from a ship, but no obvious incident of stress is necessarily required. The relatively consistent linear drift also does not imply that the drift is predictable for ac 90142 or any other ac- 9 over long time periods. Each ac-9 we have calibrated has had a unique rate of drift. Some channels drift negative and some drift positive, some approximately logarithmic and some linear, even within the same optical path. Rates of drift are currently as poor as $0.01 \mathrm{~m}^{-1}$ day $^{-1}$ in some channels in five regularly calibrated ac-9s.

The causes of instrument drift include 1) degradation of the interference filters; 2) shifts in the alignment of the optical paths due to rough handling, physical stress, and torquing on the instrument housing; 3) changing detector characteristics over time; and 4) changes in the transmissivity of the quartz pressure windows. Filter degradation accounted for most of the water offset drift observed in the early ac-9 models. Since 1995, more sensitive reference detectors and interference filters made with new optical coating technologies (MicroPlasma ${ }^{\circledR}$ deposition, OCA) have been installed in the ac-9. The longterm calibration drifts for ac90142 were recorded immediately after it had been refitted. The rates of drifts in both the absorption and attenuation channels were between $50 \%$ and $80 \%$ less after the refit. Increased instrument stability also resulted in more reproducible calibrations.

The alignment of the optical path also affects instrument drift. When deployed for in situ measurements, it is recommended that the ac- 9 be positioned vertical to $45^{\circ}$ (WET Labs, Inc. 1997), and there is evidence that it can be operated horizontally (unpublished data). Also important, however, is that water offsets change with ac- 9 orientation because the optical path becomes minutely distorted. The attenuation channels are particularly sensitive to these small changes in optical path alignment. As a result, the ac-9 cannot be calibrated vertically and then used at $45^{\circ}$ in the field, for example, because the water offset drifts will be incorrect. The magnitude of the error can be as high as $0.01 \mathrm{~m}^{-1}$.

The same holds for torquing the ac-9, where the housing on the bottom of the instrument is twisted relative to the housing on the top. This also causes changes in optical path alignment. A common application where torquing occurs is when the ac-9 is secured with hose clamps to a cage for deployment. Although the ac-9 orientation when it was calibrated and deployed may have been the same, calibration-derived water offsets will not be applicable because the instrument is being torqued relative to when it was calibrated. The best solution to problems in maintaining optical alignment is to calibrate the ac- 9 with pure water after it has been secured in its deployment cage.

A final issue in instrument drift is the internal temperature correction. The constant $K_{t}$ will inevitably drift as the other ac- 9 component characteristics change over time. Although the magnitude of the correction is usually greater than $0.010 \mathrm{~m}^{-1}$, the changes in $K_{t}$ between WET Labs calibrations a few months apart have always been small (1\%-2\%). As a result, changes in the internal temperature correction between calibrations have been less than $0.001 \mathrm{~m}^{-1}$. More importantly, WET Labs allows a deviation from the linear fit up to $0.010 \mathrm{~m}^{-1}$ for the internal temperature correction. This allowance is large relative to the precision in the raw signal. Conceivably, then, the error between two identical absorption measurements made with a meter at two different internal temperatures could be as much as $0.010 \mathrm{~m}^{-1}$. Which measurement was in error depends on the internal temperature of the meter when it was calibrated with pure water. The ideal situation in the field is one in which the internal temperature during calibration is within a couple degrees of the internal temperature during measurement. As a result, we now calibrate ac-9s immediately after the package is brought on deck after profiling. It is recommended that the internal temperature calibration files be obtained from WET Labs so any significant deviations from linearity can be noted and a correction applied if deemed necessary. The changing offsets with depth between the absorption channels in Fig. 7d are most likely due to errors associated with changes in the internal temperature and the correction algorithm.

\section{b. Measuring $a_{t}(\lambda), a_{g}(\lambda)$, and $a_{p}(\lambda)$ on the microscale}

Accurate, microscale sampling was necessary to fully describe the distribution and variability of IOPs through the water column. The profile from 31 May 1996 is a good example of how significant shifts in the contribution of $a_{g}$ and $a_{p}$ to total absorption can occur over short depth intervals (Fig. 10b). Just outside the 3-m particle peak, $a_{g}(440)$ and $a_{p}(440)$ were approximately equal, but in the center of the peak $a_{p}(440)$ was five times larger than $a_{g}(440)$. These rapid changes with depth affect the spectral vertical attenuation of the underwater light field because absorption and scattering directly control the diffuse attenuation of spectral irradiation with depth in the ocean via radiative transfer (Preisendorfer 1976). Accordingly, the downwelling diffuse attenuation coefficient for photosynthetically available radiation, $K_{d}(\mathrm{PAR})$, was 0.4 to $0.5 \mathrm{~m}^{-1} 1 \mathrm{~m}$ above and $1 \mathrm{~m}$ below the particle layer, but greater than $2 \mathrm{~m}^{-1}$ within the layer (unpublished data).

Rapid shifts occurring over centimeter intervals in $a_{g}$ and $a_{p}$ and their relative contributions to total absorption also demonstrate the importance of microscale sampling in the estimation of bulk biological and biochemical properties. An example in remote sensing is in the development of empirical relationships between in situ phytoplankton pigment absorption or CDOM absorption and spectral water-leaving radiance in coastal waters (Carder et al. 1991). Significant microscale vertical 
structure must be taken into account to accurately represent the integrated affects of water column constituents on surface reflectance. This is highlighted in the 15 June 1995 and 31 May 1996 profiles, where the most vertically heterogeneous region of the water column for $a_{g}$ and $a_{p}$ was the surface $5 \mathrm{~m}$. For these waters, this is where approximately $99 \%$ of surface reflectance originates. Additionally, since $a_{g}$ and $a_{t}$ are used to estimate $a_{p}$, the microscale distribution of $a_{g}$ and $a_{t}$ must be measured simultaneously.

\section{Summary}

Real-time in situ absorption spectra in the visible domain were replicated between different ac-9s within $\pm 0.005 \mathrm{~m}^{-1}$. This sensitivity is sufficient to resolve naturally occurring CDOM and total absorption in coastal and oceanic regions. Accurate results were only obtained, however, after water calibrations were performed to correct for instrument drift and after temperature, salinity, and scattering corrections were applied to raw absorption data. The $a_{g}(\lambda)$ and $a_{p}(\lambda)$ spectral relationships compared well with laboratory measurements. With this methodology, accurate distributions of $a_{g}(\lambda)$, $a_{t}(\lambda), a_{p}(\lambda)$, and $s$ were measured with a vertical resolution of centimeters in concert with simultaneous measurements of microscale hydrographic structure.

Acknowledgments. The authors thank W. Scott Pegau for many insightful discussions on the ac-9. An anonymous reviewer provided helpful comments on the manuscript. Additional thanks to Casey Moore and Jon Kenneke at WET Labs. We also acknowledge Brian Heikus, Alfred Hanson, and Dana Kester for laboratory expertise and materials. Finally, thanks to Jan Rines and Bob Vaillancourt for field assistance and for providing the cultures. This work was funded in part by ONR Biological/Chemical Oceanography Grant N000149510225 and NSF Grant OCE9108527.

\section{REFERENCES}

Bricaud, A., A. Morel, and L. Prieur, 1981: Absorption by dissolved organic matter in the sea (yellow substance) in the UV and visible domains. Limnol. Oceanogr., 28, 43-53.

- M. Babin, A. Morel, and H. Claustre, 1995: Variability in the chlorophyll-specific absorption coefficients of natural phytoplankton: Analysis and parameterization. J. Geophys. Res., 100, 13 321-13 332.

Carder, K. L., R. G. Steward, G. R. Harvey, and P. B. Ortner, 1989: Marine humic and fulvic acids: Their effects on remote sensing of ocean chlorophyll. Limnol. Oceanogr., 34, 68-81.

- S. K. Hawes, K. A. Baker, R. C. Smith, R. G. Steward, and B. G. Mitchell, 1991: Reflectance model for quantifying chlorophyll $\mathrm{a}$ in the presence of productivity degradation products. J. Geophys. Res., 96, 20 599-20 611.

Cowles, T. J., and R. A. Desiderio, 1993: Resolution of biological microstructure through in situ fluorescence emission spectra. Oceanogr., 6, 105-111.

Davies-Colley, R. J., and W. N. Vant, 1987: Absorption of light by yellow substance in freshwater lakes. Limnol. Oceanogr., 32, 416-425.

Donaghay, P. L., H. M. Rines, J. M. Sieburth, 1992: Simultaneous sampling of fine scale biological, chemical, and physical structure in stratified waters. Arch. Hydrobiol. Beih., 36, 97-108.

- M. S. Twardowski, J. M. Sullivan, J. E. B. Rines, and A. K. Hanson, 1996: Optical layers in an isotropic coastal ocean: An ephemeral phenomena or an inappropriate physical model? Eos, Trans. Amer. Geophys. Union, 76, 104.

Iturriaga, R., and D. A. Siegel, 1989: Microphotometric characterization of phytoplankton and detrital absorption properties in the Sargasso Sea. Limnol. Oceanogr., 34, 1706-1726.

Kirk, J. T. O., 1994: Light and Photosynthesis in Aquatic Ecosystems. $2 \mathrm{~d}$ ed. Cambridge University Press, $509 \mathrm{pp}$.

Mitchell, B. G., and D. A. Kiefer, 1988: Chlorophyll a specific absorption and fluorescence excitation spectra for light-limited phytoplankton. Deep-Sea Res., 35, 639-663.

Moore, C. M., 1994: In situ, biochemical, oceanic, optical meters: Spectral absorption, attenuation, fluorescence meters-A new window of opportunity for ocean scientists. Sea Technol., 35, $10-16$.

_ J. R. V. Zaneveld, and J. C. Kitchen, 1992: Preliminary results from an in situ spectral absorption meter. Proc. SPIE, Ocean Optics XI, 1750, 330-337.

Pegau, W. S., and J. R. V. Zaneveld, 1993: Temperature-dependent absorption of water in the red and near-infrared portions of the spectrum. Limnol. Oceanogr., 38, 188-192.

- , and — 1994: Temperature dependence of the absorption coefficient of pure water in the visible portion of the spectrum. Proc. SPIE, Ocean Optics XII, 2258, 597-604.

—, D. Gray, and J. R. V. Zaneveld, 1997: Absorption and attenuation of visible and near-infrared light in water: Dependence on temperature and salinity. Appl. Opt., 36, 6035-6046.

Preisendorfer, R. W., 1976: Hydrologic Optics. Vol. 1. U.S. Department of Commerce, $218 \mathrm{pp}$.

Sullivan, S. A., 1963: Experimental study of the absorption in distilled water, artificial sea water, and heavy water in the visible region of the spectrum. J. Opt. Soc. Amer., 53, 962-968.

Twardowski, M. S., 1998: The finescale distribution, origin, and photobleaching of the spectral absorption of dissolved organic matter in coastal waters. Ph.D. dissertation, University of Rhode Island, $289 \mathrm{pp}$.

, J. M. Sullivan, and P. L. Donaghay, 1996: Spectral dissolved absorption measurements in the visible domain: Resolving fine scale structure and dynamics in time and space. Eos, Trans. Amer. Geophys. Union, 76, 94.

WET Labs, Inc., 1997: ac-9 documentation and manual. Western Environmental Technology Laboratories, Inc., 24 pp. [Available from Environmental Technology Laboratories, Inc., 620 Applegate, Philomath, OR 97370.]

Yentsch, C. S., 1957: A non-extractive method for the quantitative estimation of chlorophyll in algal cultures. Nature, 179, 13021304.

- 1960: The influence of phytoplankton pigments on the color of sea water. Deep-Sea Res., 7, 1-9.

_ 1962: Measurement of visible light absorption by particulate matter in the ocean. Limnol. Oceanogr., 7, 207-217.

Zaneveld, J. R. V., and R. Bartz, 1984: Beam attenuation and absorption meters. Proc. SPIE, Ocean Optics VII, 489, 318-324.

, — , and J. C. Kitchen, 1990: Reflective-tube absorption meter. Proc. SPIE, Ocean Optics X, 1302, 124-136.

— J. C. Kitchen, A. Bricaud, and C. M. Moore, 1992: Analysis of in situ spectral absorption meter data. Proc. SPIE, Ocean Optics XI, 1750, 187-200.

,-- _ and C. M. Moore, 1994: The scattering error correction of reflecting-tube absorption meters. Proc. SPIE, Ocean Optics XII, 2258, 44-55.

Zepp, R. G., and P. F. Schlotzhauer, 1981: Comparison of photochemical behavior of various humic substances in water: 3 . Spectroscopic properties of humic substances. Chemosphere, 10, 479-486. 